\title{
How Much Should the NHS Pay for a QALY?
}

\author{
Alan Haycox
}

Published online: 1 May 2013

(c) Springer International Publishing Switzerland 2013

What is the purpose of the cost-effectiveness threshold employed by NICE and how do we optimise the decision criteria on which it is based? In this issue of Pharmacoeconomics, Refoios Camejo et al. (1) argue for a more dynamic approach that adjusts the threshold so as to reward innovation and prevent placing a 'dead hand' on innovation in important therapeutic areas. High levels of innovation in any therapeutic area lead to 'standard care' (the comparator for any new innovation) becoming more efficient and a reduction in unmet need. In such circumstances, the existence of a constant ICER threshold reduces the incentive for pharmaceutical companies to innovate as the clinical effectiveness of existing therapies leaves less room for additional health gains from drug innovations.

Should the threshold ICER be raised in certain disease areas to prevent the 'stifling' of innovation in areas where there has already been significant technological progress? This suggestion adds to a dazzling array of arguments that have been made for varying the cost-effectiveness threshold to advantage certain therapeutic areas. However, one compelling argument remains for maintaining a single threshold-it is the only way to maximise population health gains from a fixed healthcare budget. By equalising marginal rates of return in each therapeutic area, the health gain provided to the entire patient population from the limited healthcare resources available is maximised. The optimal funding rule under a budget constraint is to equalise marginal benefit derived from each area of investment. It would require an overwhelming argument to move away from such an optimum structure of resource

\footnotetext{
A. Haycox $(\square)$

University of Liverpool Management School,

Chatham Street, Liverpool L69 7ZH, UK

e-mail: ahay@liv.ac.uk
}

allocation given that any such move would inevitably reduce the health gain provided to the patients that we serve. I have yet to see such an 'overwhelming argument'.

Recent work from York (2) has generated an empirical estimate of the level at which the cost-effectiveness threshold should be established in the UK. Irrespective of the level at which it is set, a single cost-effectiveness threshold ensures that the scarce healthcare resources available are allocated in such a way so as to maximise health gain. Such a threshold provides a level playing field for each therapeutic area (mental illness being evaluated alongside oncology purely on the basis of potential health gain), providing clear and unambiguous signals to the pharmaceutical industry concerning the decision criteria on which the value of their drugs will be assessed and hence the extent of the potential market for any drug. The industry should not expect the health service to alter their thresholds simply as a consequence of their unwillingness to kill off an unnecessary and unwanted drug.

If we accept the population health benefits that would be likely to arise from a constant cost-effectiveness threshold, then at what level should such a threshold be set? The ICER threshold represents the 'shadow price', which captures the benefits foregone as a consequence of resources being pre-empted by NICE decision making. The exact value that should be placed on such a threshold has been the source of much debate with persuasive arguments being deployed for both an increase (3) and a decrease (4) in the threshold. Arguments for decreasing the threshold tend to emphasise the difficulties being experienced in implementing NICE guidance at the current threshold and how such difficulties will be enhanced by the stringent resource limitations being enforced on the NHS. Arguments for increasing the threshold tend to emphasise the significant increases in resources, productivity and inflation that have 
been experienced by the NHS since the threshold was initially established, which is held to justify a significant increase in the current value of the threshold.

NICE itself has been reluctant to specify a single costeffectiveness threshold on the basis that society would wish a wide variety of factors in addition to cost effectiveness to underpin healthcare resource allocation and to determine the value placed on any new intervention. Currently, NICE uses a threshold range of between $£ 20,000$ and $£ 30,000$ per QALY for 'normal' drugs and $£ 50,000$ per QALY (under strictly controlled circumstances) for drugs at the end of life.

So why is the threshold level so crucial, surely a higher level would be beneficial as NICE would introduce more drugs into the NHS? Such an argument ignores one simple concept of enormous significance to health economistsopportunity cost! The mandatory nature of NICE guidance means that any positive guidance obliges the NHS to utilise the drug in all appropriate circumstances. The resources required to facilitate this are not provided de novo by NICE but inevitably displace existing services through disinvestment or displace service developments that were being prioritised by local decision makers. The 'top slicing' of resources to support positive NICE guidance inevitably imposes significant health losses on the 'invisible' patients whose services are displaced. Obviously, it is to be hoped that the services displaced support the patients who are the least cost-effective patient group currently being treated in the local health economy. However, a more likely occurance is that the services displaced will simply represent those that lack strong and coherent local support to fight their case for continued funding. These are likely to be services for the elderly or mentally ill, which perhaps represent some of the most valuable health interventions provided to the local population. The value of such lost interventions is likely to be enhanced by cutbacks in social services, which will further reduce the availability of support to these patient groups. In this manner, positive NICE guidance acts as a reduction in the discretionary budget that is available to local decision makers to invest in local priority areas. The greater the budgetary implications of the NICE guidance then consequently the greater will be the displacement of local discretion and, in current circumstances, the less likely will local social services be able to replace any displaced services.

In order for NICE decision making to contribute to the efficient use of NHS resources, a positive NICE decision should only be made when the health benefits derived from a new drug or intervention exceed the benefits foregone through the activity being displaced or the 'next best alternative' investment opportunity at the local level. Obviously, the health benefits 'foregone' will be locally and context specific and hence the cost-effectiveness threshold represents an estimate of the average opportunity cost imposed on the NHS as a consequence of the loss of one QALY through the preempting of resources by NICE decision making. Thus, in evaluating new interventions NICE must dispassionately and objectively assess the extent to which the very visible health gains derived from the new service being evaluated would outweigh the perhaps less visible but no less real opportunity costs arising at the local level, as a consequence of the health losses resulting from the unavailability of resources to local health care decision makers in what is increasingly becoming a zero sum game.

NICE was established to ensure the maintenance of a national health service in which the quality and nature of patient care received was not dependent on the patient's postcode. In this regard NICE has achieved much in its comparatively short life in providing a 'level playing field' for both patients and the pharmaceutical industry. The assessment process has led to significant improvements in both the overall efficiency of health care provision and (perhaps even more importantly) in terms of equalising patient access to services. However, it is important to acknowledge that with great power comes great responsibility. Those who pre-empt the use of healthcare resources must ensure that their decisions take adequate account of the health losses that will inevitably arise through the health loss imposed on 'invisible' local patients. In this regard, it is vital that the introduction of value-based pricing in the UK does not involve the dismantling of a single threshold in favour of a less transparent system that perhaps would be more open to more overt political manipulation. It is the role of HTA systems to optimise the quality and quantity of healthcare provided to their populations irrespective of which interest groups are discomforted in the process.

The existence of a fixed and widely acknowledged costeffectiveness threshold enables the health services to engage in informed negotiations concerning the linking of price to the clinical value of any new service. Being an informed consumer with rules that are easily understandable and fair to all suppliers ensures a level playing field in resource allocation both over time and between disease areas. It ensures that the drug development process responds appropriately to the expressed needs of the health service and that 'true' innovation in areas of greatest need is appropriately rewarded. The drug development process can only be helped by having a well-informed consumer who effectively communicates their decision criteria in a well-functioning market place-and this is what a common cost-effectiveness threshold provides.

Conflict of interest Dr. Haycox is a member of NICE Appraisal board ' $C$ ' but has no other conflicts. The views and opinions expressed in this editorial are entirely his own and should not be interpreted as being in any way sanctioned or condoned by NICE. 


\section{References}

1. Refoios Camejo R, McGrath C, Miraldo M, Rutten F. The determinants of cost-effectiveness potential: an historical perspective on lipid-lowering therapies. Pharmacoeconomics. 2013. doi:10.1007/s40273-013-0041-x.
2. Claxton $\mathrm{K}$ et al. 'Methods for the estimation of the NICE costeffectiveness threshold' CHE Research Paper 81, 2013.

3. Towse A. 'Should NICE's threshold range for cost per QALY be raised? Yes' BMJ. 2009;338:b181.

4. Rafferty J. 'Should NICE's threshold range for cost per QALY be raised? No’ BMJ. 2009;338:b185. 